\title{
Methylation Quantitative Trait Loci are Largely Consistent across Disease States in Crohn's disease
}

Suresh Venkateswaran ${ }^{1 *}$, Hari K Somineni ${ }^{1,2}$, Varun Kilaru ${ }^{3}$, Seyma Katrinli ${ }^{3}$, Jarod Prince ${ }^{1}$, David T

Okou $^{1}$, Jeffrey S Hyams ${ }^{5}$, Lee A Denson ${ }^{6}$, Richard Kellermayer ${ }^{7}$, Greg Gibson ${ }^{8}$, David J Cutler ${ }^{9}$, Alicia K Smith $^{3,4}$, Subra Kugathasan ${ }^{1,9}$ Karen N Conneely ${ }^{\$ \%} *$

\section{Author Affiliations}

${ }^{1}$ Division of Pediatric Gastroenterology, Department of Pediatrics, Emory University School of Medicine \& Children's Healthcare of Atlanta, Atlanta, GA, USA

${ }^{2}$ Genetics and Molecular Biology Program, Emory University, Atlanta, GA, USA

${ }^{3}$ Department of Gynecology \& Obstetrics, Emory University School of Medicine, Atlanta, GA, USA

${ }^{4}$ Department of Psychiatry \& Behavioral Sciences, Emory University, Atlanta, GA, USA

${ }^{5}$ Division of Digestive Diseases, Hepatology, and Nutrition, Connecticut Children's Medical Center, Hartford, CT, USA

${ }^{6}$ Division of Pediatric Gastroenterology, Hepatology, and Nutrition, Cincinnati Children's Hospital Medical Center, Cincinnati, $\mathrm{OH}$, USA

${ }^{7}$ Section of Pediatric Gastroenterology, Texas Children's Hospital Baylor College of Medicine, Houston, TX, USA

${ }^{8}$ Center for Integrative Genomics, Georgia Institute of Technology, Atlanta, GA, USA

${ }^{9}$ Department of Human Genetics, Emory University, Atlanta, GA, USA

\$ Equal contribution for last authorship

*Corresponding authors: $\quad$ Karen N Conneely, $\mathrm{PhD}$

Department of Human Genetics, Emory University,

Atlanta, GA, USA

Tel: 404-727-2986

e-mail: kconnee@emory.edu

Suresh Venkateswaran, $\mathrm{PhD}$

Department of Pediatrics,

Emory University,

Atlanta, GA, USA

Tel: 404-727-1455

e-mail: svenk30@emory.edu 


\begin{abstract}
Background

In a recent study, we identified $1189 \mathrm{CpG}$ sites whose DNA methylation (DNAm) level in blood distinguished Crohn's disease (CD) cases from controls. We also demonstrated that the vast majority of these differences were a consequence of disease, rather than a cause of CD. Since methylation can be influenced by both genetic and environmental factors, here we focus on CpGs under demonstrable genetic control (methylation quantitative trait loci, or mQTLs). By comparing mQTL patterns across disease states and tissue (blood vs. ileum), we may distinguish patterns unique to CD. Such DNAm patterns may be relevant for the developmental origins of $\mathrm{CD}$.
\end{abstract}

\title{
Methods
}

We investigated three datasets: (i) 402 blood samples from 164 newly diagnosed pediatric CD patients taken at two time points, and 74 non-IBD controls (ii) 780 blood samples from a non-CD adult population and (iii) 40 ileal biopsies (17 CD cases and 23 non-IBD controls) from group (i). Genome-wide DNAm profiling and genotyping were performed using the Illumina MethylationEPIC and Illumina Multi-Ethnic arrays. SNP-CpG associations were tested via linear models adjusted for age, gender, disease status, disease subtype, estimated cell type and three genotype-based principal components. We used a Bonferroni-adjusted significance threshold to identify significantly associated SNP-CpG pairs, but also considered larger sets identified by a false discovery rate criterion

\section{Results}

In total, we observed 535,448 SNP-CpG associations between 287,881 SNPs and 12,843 CpG sites $\left(P<8.21 \times 10^{-14}\right)$. These associations and their effects are highly consistent across different ages, races, disease states, and tissue types, suggesting that the vast majority of these mQTLs participate in common gene regulation. However, genes near CpGs associated with IBD SNPs were enriched for 18 KEGG 
pathways relevant to IBD-linked immune function and inflammatory responses. We observed suggestive evidence for a small number of tissue-specific associations and disease-specific ileal associations in ileum, though larger studies will be needed to confirm these results.

\section{Conclusion}

The vast majority of blood derived mQTLs are commonly shared across individuals. However, we have identified a subset of such, which may be involved in processes related to CD. Independent cohort studies will be required to validate these findings.

Keywords: Methylation quantitative trait loci (mQTL), Inflammatory Bowel Disease (IBD), Crohn's Disease (CD), DNA methylation (DNAm)

\section{Background}

A majority of inflammatory bowel disease (IBD) trait-associated genomic variants identified in genomewide association studies (GWAS) reside in the genome's non-coding regions [1]. In the past decade, studies have focused on demonstrating the potential of these variants to influence specific traits or diseases through transcriptomic regulation [2-4]. Previous expression quantitative trait loci (eQTL) studies in IBD proposed that the genetic influences on gene expression are tissue-specific [5], diseasespecific [6], and inflammation-specific [5, 7]. Meanwhile, recent advances in high-throughput array technologies have enabled genome-wide DNA methylation (DNAm) profiling for $>850,000$ CpGs. DNAm is one of the primary epigenetic mechanisms involved in gene expression regulation. Investigation of DNAm has the potential to advance our understanding of complex disease pathogenesis $[8,9]$. In an epigenome-wide association study (EWAS), we recently identified 1189 CpGs showing differential DNAm in Crohn's disease (CD) patients at diagnosis, compared to non-IBD controls [10]. A Mendelian Randomization (MR) approach revealed that most of the DNAm changes observed in CD patients are consequences of the disease rather than causal factors influencing disease risk [10]. Since DNAm can be 
influenced by both environmental and genetic factors, in this paper we will focus on identification of methylation quantitative loci (mQTLs), or SNPs that associate with DNAm at specific CpG sites. We will compare mQTLs found within blood across disease state and progression, as well as age and race. Finally, we will compare patterns found in blood to those found in ileal (disease) tissue. In general, we are looking for mQTL patterns that distinguish CD cases from CD controls, and mQTLs that are contextspecific.

Identifying SNP-CpG associations and comparing them between disease groups will provide epigenetic annotation of IBD variants, facilitating their functional characterization. The comparative mQTL study has the potential to reveal differences in interactions between genetic and epigenetic mechanisms in IBD patients vs. controls. Further, the identification of mQTLs can aid MR approaches through identification of potential instruments to distinguish the casual vs. consequential role of DNAm in complex diseases [10], and can shed light on causal relationships between gene expression and DNAm in complex diseases $[11,12]$.

Investigating whether SNP-CpG associations are stable or dynamic over the course of the diseases can help inform whether the association might be a useful biomarker for disease, a useful marker for treatment, a potential cause of disease, or without obvious utility. A recent longitudinal analysis at five different stages showed that blood mQTLs are generally stable across the life span [13]. However, among the 1189 differentially methylated CpG sites identified in our previous study from blood derived DNA, we observed that during the CD disease course (at 1-3 years follow-up), DNAm patterns in cases reverted back to levels observed in healthy controls. [10]. We also noted high correlations between DNAm at these 1189 sites and levels of the inflammatory marker C-reactive protein (CRP) [10]. Investigation of mQTLs in CD patients pre- and post-treatment will reveal whether the genetic influence on DNAm at these sites varies over the disease course. 
CD commonly affects the terminal ileum. Thus, in contrast to DNAm patterns in blood, methylation patterns in the ileum might be considered patterns seen at the "site of disease". Consequently, ileal DNAm is more likely to be causative, compared to patterns observed in other tissues. Thus, we examine ileum specific mQTL associations in CD patients.

Here, we used blood samples obtained from a total of 238 pediatric subjects; 164 patients with CD and 74 healthy controls sampled from the Risk Stratification and Identification of Immunogenetics and Microbial Markers of Rapid Disease Progression in Children with Crohn's Disease (RISK) study [14]. We replicated our analysis in an independent cohort of 780 blood samples obtained from prospectivelyrecruited adults in the Grady Trauma Project (GTP). Enrichment analysis showed that the previouslyidentified IBD GWAS SNPs are significantly enriched for mQTLs in the CD cohort, but not in the adult cohort. To assess whether the mQTLs are specific to disease, disease course, or tissue type, we performed separate mQTL analyses in $164 \mathrm{CD}$ cases at diagnosis, in cases at follow-up (1-3 years from the day of diagnosis), in 74 healthy controls and in 40 ileal biopsies (obtained from a subset of subjects included in the blood mQTL analysis; 23 controls and 17 patients with CD at diagnosis).

\section{Results}

\section{Blood mQTL discovery}

To identify blood mQTLs, we tested the association between 3,109,863 SNPs and 609,192 CpG sites from 238 blood samples taken from 164 pediatric patients with CD at diagnosis and 74 matched non-IBD controls (Additional file 1: Table S1). We identified 535,448 associated SNP-CpG pairs involving 303,986 (9.8\%) unique SNPs and 13,823 (2.3\%) unique CpGs, at a Bonferroni adjusted genome-wide significance of $P<8.21 \times 10^{-14}$ (Fig. 1A). On average, each $\mathrm{CpG}$ site was associated with $\sim 38$ SNPs (interquartile range (IQR): 4 - 42), which likely reflects linkage disequilibrium (LD) between proximal 
SNPs, while each mQTL variant associated with 1.73 CpGs (IQR: 1 to 2). Using the clump command in PLINK [15], we identified 16,313 LD pruned proximal SNPs that were associated with 13,202 CpG sites, which made a total of 24,523 associations.

93\% $(n=498,261)$ of the SNP-CpG pairs involved associations between a genetic variant and CpG site within $1 \mathrm{MB}( \pm 500 \mathrm{~kb}$; Fig. 1B) and were considered cis-acting. The remaining $7 \%$ appeared to be transacting $(n=37,187)$; of these, 44\% $(n=16,457)$ involved CpGs and SNPs from the same chromosome that were $>500 \mathrm{~kb}$ apart, while 56\% $(\mathrm{n}=20,730)$ involved a SNP and CpG from different chromosomes (Fig. 1C). On average, cis-acting mQTLs had larger effects with a mean effect size of 8.7\% (IQR: $5.3-10.8$ ) change in DNAm per allele, compared to trans-acting mQTLs with a mean effect size of $6.9 \%$ (IQR: 2.8 - 9.4) change in DNAm per allele (Mann-Whitney-Wilcoxon $P<2.2 \times 10^{-16}$. (Figure S1). Similarly, on average cis-acting mQTLs had larger effect sizes, with a mean effect size of 0.0046 (IQR: $-0.06-0.07$; SD: 0.097), compared to trans-acting mQTLs, with a mean effect size of 0.0035 (IQR: $-0.06-0.053$; SD: 0.081) (Figure S1). A list of all cis- and trans- SNP-CpG pairs identified from 238 blood samples is provided in Additional file 2: Table S2. Enrichment analysis of cis-mQTL associated SNPs $(n=287,881)$ showed that they are more likely than other SNPs on the array to occur $1 \mathrm{~kb}$ upstream of transcription start site (TSS), $1 \mathrm{~kb}$ downstream of transcription end site (TES), in exons, the 3' or 5' untranslated regions, or within 2 bp of a splicing junction $\left(P<2 \times 10^{-10}\right.$; Additional file 1: Table S3). Similarly, cismQTL associated CpGs $(n=12,843)$ are more likely than other CpGs on the array to occur in CpG islands and shores $\left(P<2.2 \times 10^{-16}\right.$; Additional file 1: Tables S4-5.)

\section{Blood mQTLs are highly replicable in an independent dataset regardless of age and race}

To evaluate whether our blood mQTLs are unique to IBD, or more common to the human condition, we tested all cis associations in an independent cohort of 780 blood samples (GTP cohort) collected previously [16]. More details about demographic information of this dataset are available in Almli et al. [16] as well as in our methods section. Notably, while our discovery dataset consists of pediatric patients 
with predominantly Caucasian ancestry (78\%), the GTP dataset is composed of prospectively-recruited adult individuals with predominantly African American ancestry (93\%), with a mean age of 42.26 (SD: 12.31) years (Additional file 1: Table S1). After QC, 5,971,966 genomic variants and 608,245 CpG sites were retained for 780 blood samples. To test for the replicability of blood mQTLs from the discovery cohort, we focused only on the SNPs and CpG sites identified in the discovery cohort. In total, 253,000 out of 287,881 SNPs $(87.9 \%)$ and 12,808 out of $12,843 \mathrm{CpG}$ sites $(99.7 \%)$ were available to be tested in the replication cohort, representing a total of 425,288 CpG-SNP relationships. Applying a standardized genome-wide significance threshold of $P<5 \times 10^{-8}$, we replicated $\sim 84 \%$ of the $\mathrm{CD}$ dataset associations (358,804 SNP-CpG pairs out of 425,288 tested). This includes $87 \%(n=220,468)$ of SNPs, and $85 \%$ $(\mathrm{n}=10,905)$ of $\mathrm{CpG}$ sites. At a less stringent nominal significance threshold of $P<0.05$, we detected 388,609 associations, of which $91.3 \%(n=388,217)$ were considered replications, where the effect sizes of both CD and GTP cohort associations are directionally consistent. Test statistics for these associations were highly correlated in the discovery vs. replication cohorts $\left(\mathrm{R}^{2}=0.94, P<2.2 \times 10^{-16}\right.$; Fig. 2), indicating that most blood SNP-CpG associations are consistent regardless of age, race and disease status. Summary statistics for the 388,609 SNP-CpG associations are provided in Additional file 3: Table S6. Among the few mQTLs showing associations in the opposite direction $(n=393 ; 358$ unique SNPs and 119 CpG sites) between the CD and GTP cohorts (i.e. a SNP associated with increased methylation in one cohort, and decreased methylation of the same $\mathrm{CpG}$ in another cohort), we did not observe significant enrichment for any KEGG pathways, including inflammation or immune-related pathways (data not shown). Similarly, none of the previously identified IBD-associated SNPs $[17,18]$ or CD-associated CpGs [10] were involved in these 393 mQTL associations. These results are consistent with our previous [19] findings that blood mQTLs are stable across ancestries (African American vs. Caucasian), and developmental stages (neonate vs. adult). Collectively, our results indicate that the vast majority of genetic influence on changes in blood DNAm are highly consistent regardless of disease, race and age groups, and thus unlikely to be casually related to $\mathrm{CD}$ in any substantial way. 


\section{CD-specific mQTLs in blood samples}

To assess whether mQTLs vary with respect to disease status, we retested each of the 498,261 significant SNP-CpG pairs separately within controls $(n=74)$ and within CD cases $(n=164)$. We observed a strong positive correlation $\left(\mathrm{R}^{2}=0.97, P<2.2 \times 10^{-16}\right)$ between effect sizes estimated from controls vs. cases (Fig. 3A). Collectively, our data establish that while methylation levels may vary with $\mathrm{CD}$ at specific sites (CD-associated CpG sites) [10], the influence of SNPs on CpG methylation levels at CpG sites remains consistent in the presence or absence of disease. Results obtained for CD cases and healthy controls are provided in Additional file 4: Table S7.

\section{Longitudinal profiling of mQTLs}

In our previous report, we demonstrated that with treatment, DNAm changes associated with CD at diagnosis revert to the levels seen in healthy controls [10]. To examine the longitudinal trajectory of SNPCpG associations and their dynamics during the course of the disease, we took advantage of our longitudinal DNAm data generated from same individuals from the discovery cohort (164 CD cases at diagnosis vs. follow-up). We found that mQTL effect sizes were similar between diagnosis and at 3-year follow-up in $\mathrm{CD}$ patients, showing a strong positive correlation in magnitude and direction of effect $\left(\mathrm{R}^{2}=0.99, P<2.2 \times 10^{-16}\right.$; Fig. 3B; Additional file 4: Table S7). All discovery SNP-CpG associations $(\mathrm{n}=497,689)$ were nominally significant at baseline $(P<0.05)$, and $99.5 \%(\mathrm{n}=497,443)$ were nominally significant during the course of the disease $(P<0.05)$. Therefore, our results suggest that these genetic associations are independent of disease status or treatment.

\section{Tissue-specific associations between blood and ileum}

To further evaluate how the mQTLs identified in our blood-based discovery dataset act in tissues more directly relevant to disease, we estimated SNP-CpG effect sizes using DNAm data from ileal biopsies (n=40) and compared them to effects observed in blood. With the standard replication threshold of $P<$ 
0.05, we replicated 50.4\% $(\mathrm{n}=238,400)$ blood SNP-CpG associations in ileal biopsies. Overall, we noted an extremely strong correlation in effect sizes between ileal and blood samples, with $91.4 \%(n=473,382)$ of mQTLs showing directional consistency $\left(\mathrm{R}^{2}=0.83, P<2.2 \times 10^{-16}\right.$; Fig. 3D). A list of all ileal biopsy mQTL results are provided in Additional file 5: Table S8. We further investigated CpGs $(n=2,188)$ from SNP-CpG associations $(n=24,879)$ with directionally inconsistent effects between blood and biopsy for tissue-specific biological functions, and observed that these sites were more likely to localize to genes involved in metabolic pathways $\left(P=3.34 \times 10^{-8}\right.$; Table S9). Using summary statistics from two different studies, Qi et al [20] recently showed that mQTLs are highly correlated among the blood and brain tissues. Similarly, using blood and four regions of postmortem brain samples, we previously showed a strong mQTL correlation among the tissues, especially an extreme overlap within the four brain regions [19]. Our current results show a similar pattern for blood vs. ileum taken from the same patients, where the mQTL effects are highly correlated between blood and ileal biopsies.

\section{Enrichment of previously-identified IBD susceptible SNPs in blood mQTLs}

To date, large-scale GWAS meta-analysis have identified 241 susceptibility loci for IBD [17, 18]. A majority of these (>90\%) localize to non-coding regions, presenting a key challenge to identify the functional variants and the relevant genes they act upon. Of these 241 previously-identified IBD SNPs, 168 were genotyped in our discovery dataset. We found that 37 (22.2\%) of these 168 SNPs associated with $69 \mathrm{CpG}$ sites in our discovery set of mQTL associations $\left(P<8.21 \times 10^{-14}\right.$; Figure S2A; Additional file 6: Table S10). Notably, all of these associations were cis-mQTL associations: $90 \%(\mathrm{n}=62)$ of these CpGs were located within $100 \mathrm{~kb}$ from the associated GWAS variant. Of these $62 \mathrm{CpGs,} 13 \%$ are located within $1.5 \mathrm{~kb}$ of a transcriptional start site (TSS200 or TSS1500), and 47.8\% are between the start and stop codon. These results provide an additional 31 genes potentially associated with the previousidentified IBD SNPs, where the associated CpGs may be located within the gene body or near the TSS or promotor regions of the gene. Notably, 4 of the established IBD-associated SNPs (rs13407913, rs798502, rs59043219 and rs1819333) showed association with >5 CpGs located within the gene bodies of ADCY3, 
GNA12, IRF6, and RNASET2/RPS6KA2, respectively. The 37 IBD-associated SNPs comprised $.013 \%$ of the total number of mQTL SNPs observed in our discovery cohort (Additional file 6: Table S10). For comparison, in our GTP cohort, which did not include IBD cases, we observed associations between 87 IBD-associated SNPs and 252 nearby CpGs. However a greater number of mQTL associations were observed in total in this larger cohort, the 87 IBD-associated SNPs comprised a smaller percentage of the total $(0.006 \%$; Additional file 6: Table S10). This suggests that the mQTLs identified in our discovery cohort (which is 69\% IBD patients) are significantly enriched IBD-associated SNPs (OR = 2.1 [CI: 1.4 to 3.1], Fisher's exact $P=2.71 \times 10^{-4}$ ). Summary statistics for 69 and $253 \mathrm{SNP}-\mathrm{CpG}$ associations from the IBD susceptibility SNPs identified in both discovery and replication cohorts are provided in Table 1.

We further searched for previously-identified IBD SNPs in a wider set of mQTL results identified using a less stringent significance threshold $(\mathrm{FDR}<0.05)$ in the discovery cohort. At this threshold, 64.2\% $(\mathrm{n}=108)$ of IBD SNPs were associated with $453 \mathrm{CpGs}$ within $1 \mathrm{MB}$, comprising 454 cis-mQTL associations (Additional file 6: Table S10). Of these CpGs, 84.5\% ( $\mathrm{n}=384)$ were located within $100 \mathrm{~kb}$ of the associated GWAS variant (Fig. 4A). Further disease-specific analysis on these mQTLs showed that similar to SNPs across the genome, associations between IBD-associated SNP and DNAm in blood are extremely consistent between CD cases $(n=164)$ vs. controls $(n=74)$ (Fig. 4B, Additional file 6: Table S10).

\section{Pathway enrichment of CpG sites that are under the influence of IBD-associated SNPs}

To investigate the utility of DNAm as a tool to identify functional variants and underlying regulatory mechanisms that may contribute to our fundamental understanding of CD susceptibility and pathogenesis, we examined whether CpG sites associated with IBD-associated SNPs localize near genes associated with specific pathways. We first evaluated all 69 CpGs whose blood DNAm associated with IBD SNPs in the

discovery analysis $\left(P<8.21 \times 10^{-14}\right)$ to identify the novel pathways that are relevant to $\mathrm{CD}$. Our pathway enrichment analysis identified 18 KEGG pathways that were more likely to occur in the IBD SNP- 
associated CpGs than would be expected by chance (FDR < 0.05; Additional file 1: Table S11). Among these, five were relevant to immune function and other pathways, including human cytomegalovirus infection, insulin resistance, vascular smooth muscle contraction, hepatitis B, and chemokine signaling. Pathway enrichment analysis on an expanded set of $\mathrm{CpG}$ sites $(\mathrm{n}=454)$ that associated with IBD SNPs according to a looser significance threshold (FDR < 0.05) showed enrichment for 7 KEGG pathways (FDR < 0.05), including a natural killer cell mediated toxicity pathway. We also observed nominally significant $(P<0.05)$ enrichment for immune and inflammatory related pathways such as MAPK signaling pathway, cytokine-cytokine receptor interaction, inflammatory bowel disease (IBD), JAKSTAT signaling pathway, and Th17 cell differentiation (Additional file 1: Table S12).

\section{Influence of previously-identified IBD SNPs on DNAm in ileum mQTLs}

We next investigated the 37 SNPs that were both IBD-associated [17] and associated with CpGs in our discovery cohort $\left(P<8.21 \times 10^{-14}\right)$ to identify whether they were also mQTLs in ileum. Seventeen of 37 SNPs were nominally significant mQTLs $(P<0.05)$ in ileum as well, and effect sizes showed a strong positive correlation between ileum and blood $\left(\mathrm{R}^{2}=0.789, P<7.87 \times 10^{-16}\right.$, Figure S2C; Additional file 7: Table S13).

We also followed up on the 108 FDR-significant IBD-associated SNPs in ileum. We tested 370 of the 454 blood mQTL pairs for association in ileum. These mQTL pairs included 85 of the 108 IBD-associated SNPs; the remaining 23 SNPs were not tested due to insufficient genetic variation in the smaller number of ileum samples. At a nominal threshold of $P<0.05$, we detected associations for 51 of 85 IBDassociated SNPs. In total, 91 (24.6\%) of 370 blood mQTLs in ileum were nominally significant, and the effect sizes were consistent in direction for 89 of these, indicating replication (Fig. 4C; Additional file 8: Table S14). The two IBD SNPs showing association in the opposite direction in ileum (rs35320439, and rs516246) were independently associated with CpGs cg07689252 and cg26360664 (Fig. 4C). Neither of these CpGs were CD-associated CpGs. The strong positive correlation and directionally consistent effects 
$\left(\mathrm{R}^{2}=0.87 ; P<2.2 \times 10^{-16}\right.$; Fig. 4C) suggest that the IBD-associated SNPs influence the DNAm levels mostly similarly across both tissues. Notably, performing this analysis separately in CD cases $(n=17)$ and controls $(n=23)$ in ileum showed disease-specific patterns for 13 of the IBD associated SNPs (Fig. 4D; Additional file 8: Table S14). These results suggest there may be disease-specific SNP-CpG associations in actual disease-relevant tissues such as ileum for CD. However, given our smaller sample size $(\mathrm{n}=40)$ and the nominal significance level, further confirmation is needed in larger studies of disease-relevant tissues such as ileum.

\section{CD-associated differentially methylated CpGs in blood mQTLs}

Recently, we identified 1189 CD associated CpG sites whose methylation level in blood distinguished CD cases from non-IBD controls [10]. After exclusion of 212 CpGs with one or more SNPs in their probe sequence, we tested 977 of our previously-identified $\mathrm{CpG}$ sites for associations with SNPs within 1MB. In the discovery cohort, $22(1.8 \%)$ of these $977 \mathrm{CpGs}$ associated with 565 unique SNPs for a total of 565 SNP-CpG associations $\left(P<2.81 \times 10^{-14}\right.$; Figure S2B; Additional file 9: Table S15). Although we did not observe an enrichment of CD-associated CpG sites compared to the replication cohort (Table 1), one CDassociated $\mathrm{CpG}$ site $\operatorname{cg} 20406979$ had a strong association $\left(P=7.60 \times 10^{-25}\right)$ with SNP rs1819333, previously reported to associate with CD [10]. This SNP and CpG site were located 314 nucleotides apart, $3 \mathrm{~kb}$ upstream of the transcription start site for the gene RNASET2.

We further investigated the $977 \mathrm{CD}$-associated differentially methylated CpGs using an FDR-based significance threshold $($ FDR $<0.05)$ and observed a total of 7819 cis-associations. $29 \%(n=285)$ of 977 CD-associated CpGs were associated with 7,120 nearby genomic variants (Additional file 9: Table S15), with $59.9 \%$ of these CpGs associating with more than 5 SNPs (Fig. 5A). Further separate analysis on CD cases $(n=164)$ and controls $(n=74)$ indicates that the mQTLs associated with CD-associated DNAm sites in the blood are extremely consistent across the disease types (Fig. 5B; Additional file 9: Table S15). 
Next, we investigated whether the CD-associated CpGs associated with 108 previously-identified IBD risk SNPs within 1MB. We observed 2 IBD SNPs (rs1819333, rs3853824) associated with 4 CDassociated CpGs (cg20406979, cg23216724, cg15706657, and cg15815084) for a total of 4 cis associations. Of those, three CpGs cg20406979, cg23216724, and cg15706657 had stronger association with one IBD associated SNP rs $1819333\left(P=7.60 \times 10^{-25}, P=1.2 \times 10^{-11}, \& P=9.3 \times 10^{-07}\right.$, respectively; Additional file 9: Table S15).

\section{Influence of SNPs on CD-associated CpGs in Ileum mQTLs}

Next, we investigated 22 of the 1189 CpGs previously associated with CD in blood [10] that were also blood mQTLs $\left(P<8.21 \times 10^{-14}\right)$ to see if they are also mQTLs in ileum. In blood, these CpGs were involved in 565 significant SNP-CpG associations. We replicated $173(30.6 \%)$ of these associations in ileal biopsies using a nominal threshold of $P<0.05$, and observed effect sizes are consistent in magnitude and direction in ileum vs. blood $\left(\mathrm{R}^{2}=0.82 ; P<2.2 \times 10^{-16}\right.$; Figure S2D; Additional file 10: Table S16).

We performed similar comparisons for the $285 \mathrm{CD}$-associated CpGs that were significant at FDR $<.05$ in blood, which were involved in 7819 significant SNP-CpG associations in blood. We followed up on 6,227 of these SNP-CpG pairs in ileum; the remainder were not tested due to insufficient genetic variation among the 40 ileal samples. At a nominal threshold of $P<0.05$, we detected 47 of 285 significant CDassociated CpGs and 1,002 (16.1\%) out of 6,227 blood SNP-CpG pairs in ileum (P < 0.05). Among these $1002,65.4 \%(\mathrm{n}=655)$ showed directionally consistent associations $\left(\mathrm{R}^{2}=0.92 ; P<2.2 \times 10^{-16}\right.$; Fig. 5C). The 34.6\% ( $\mathrm{n}=347)$ of CpG-SNP associations that showed association in the opposite direction in blood vs. ileum (Additional file 10: Table S16) comprised 12 CD-associated CpGs that were associated with 341 SNPs. Boxplots for the top SNP associated to the 12 CD-associated CpGs are compared between blood and ileum (Additional file 10: Table S16). None of the IBD SNPs showed the opposite mQTL effects between blood and ileum. Overall, only one IBD SNP (rs1819333) was nominally associated with a CD-associated CpG $(\operatorname{cg} 15706657)$ in ileum $(P=0.040)$, and the association was directionally consistent 
with blood results in our discovery cohort. Our analysis stratified by CD suggested possibly distinct effects for cases $(n=17)$ vs. controls $(n=23)$ in 120 mQTLs (115 SNPs and 13 CD-associated CpGs) in ileum (Fig. 5D, Additional file 11: Table S17), though a much larger sample would be required to test this formally.

\section{Discussion}

The primary focus of this study was to identify and characterize mQTLs in blood from 238 pediatric samples of European ancestry. Our findings establish that blood mQTLs are robust and reproducible, supporting their generalizability across age groups, ancestries, disease status, and DNA sample source, and offer a valuable source for future studies examining blood mQTLs. Recent studies have identified different sets of differential DNAm patterns in various tissue types [21, 22], and previous epigenomic and transcriptomic studies in IBD suggest that disease-relevant tissue samples are needed to develop precise biomarkers for the disease subtypes [5, 23-25]. Our findings show that among genome-wide-significant mQTLs, genetic influences on DNAm are generally consistent across blood and ileum, even among IBD SNPs and CD-associated CpG sites. However, we observed lower cross-tissue reproducibility using a relaxed FDR-based significance criterion. Using a threshold of FDR $<0.05$ in blood and nominal association $(\mathrm{P}<.05)$ in ileum, we identified two IBD SNPs (Figure 4C) and 12 CD-associated CpGs (Figure 5C; Figure S3) with associations in the opposite direction between blood and ileum. This could suggest that a small portion of mQTLs act in a tissue-specific manner, but could also be due to the low power and relaxed significance criteria in the ileal analysis. Studies with large numbers of samples from both blood and ileal biopsies from the same cohort are needed to formally test for tissue-specific associations for the previously-identified IBD SNPs or CD-associated CpGs.

Blood mQTLs were also consistent in comparisons of CD patients pre- vs. post-treatment and in $\mathrm{CD}$ patients compared to healthy matched controls or to an external epidemiologic sample of predominantly African American adults. This trend was observed even when we focused on previously-identified IBD 
SNPs and CD-associated CpGs, though was not as clear when studied in the ileal biopsies, again likely due to the small numbers.

Our study has several limitations. We did not explore associations involving rare variants in this study given limited power to identify such associations. In particular, because of the limited number of ileal samples used in this study, power was limited to identify tissue-specific mQTLs or to detect functionally important associations involving previously-identified IBD variants in the disease-relevant ileum.

\section{Conclusions}

We performed the first large mQTL study in the IBD research community that allows comparisons preand post-treatment, between disease groups, and between blood and ileum sampled from the same patients. The vast majority of blood derived mQTLs are commonly shared across individuals. Also, our study showed a smaller portion of tissue- and disease-specific SNP-CpG associations for previouslyidentified IBD-associated SNPs or CD-associated CpGs in the blood. In contrast, such associations in ileum are not clear, warranting further study in disease relevant tissue samples from a larger cohort.

\section{Methods}

Study Population and design of discovery cohort: The discovery cohort was a subset of pediatric subjects recruited under the Risk Stratification and Identification of Immunogenetic and Microbial Markers of Rapid Disease Progression in Children with Crohn's Disease (RISK) study [14] for whom DNAm data had been collected. A detailed description of the dataset is provided in our previous study [10]. In total, 164 newly diagnosed, pediatric patients with Crohn's disease (cases) at two time-points (diagnosis and 3-year follow-up) and 74 non-IBD controls had DNAm measured for this study. Patients 
with no bowel pathology upon endoscopy, negative gut inflammation, and continued presentation as asymptomatic for IBD during follow-up were considered as non-IBD controls.

Blood genotype data for CD cohort: Peripheral blood DNA genotypes were obtained for 238 subjects using Infinium Multi-Ethnic Global-8 Kit (Illumina, San Diego, CA) and processed with GenomeStudio software. Detailed quality control (QC) steps for both samples and variants are provided in our earlier study [10]. Briefly, sample QC was performed by checking that (i) genotype call rates $>95 \%$, (ii) inferred gender is consistent with clinical records, and (iii) subjects are unrelated according to a pairwise identityby-descent test. Similarly, SNP QC was performed by identifying and removing SNPs with low call rate ( $<95 \%)$, (ii) Hardy-Weinberg disequilibrium $\left(P<1.0 \times 10^{-3}\right)$, or (iii) minor allele frequency (MAF) $<5 \%$, as well as (iv) non-autosomal variants and (v) variants mapping to multiple locations. This resulted in a discovery data set consisting of 636,006 high-quality variants. These variants for all 238 subjects were then used to impute genotypes from the 1000 Genomes Project Phase 3 autosomal reference panel, using IMPUTE2 software [26]. Imputed SNPs with MAF<1\% and imputation quality scores <80\% were removed. We also removed imputed variants with no dbSNP annotation, INDELs/CNV, and SNPs with missing genotype information in $>5 \%$ of the samples. Lastly, SNPs with $<5$ individuals in each group (homologous reference (AA), heterozygous (AB), and homologous variant (BB)) were excluded. In total, 3,109,863 high-quality SNPs for all 238 subjects were retained across the entire genome.

Blood and Ileal DNA methylation data for CD cohort: DNAm data was profiled for 402 samples (164 CD cases at diagnosis and 3-years follow-up and 74 healthy controls at diagnosis) as described in our previous study [10]. In this study, we have added DNAm data for 40 ileal biopsies, performed in $17 \mathrm{CD}$ cases and 23 non-IBD controls from the discovery cohort. Genome-wide DNA methylation for these samples was profiled at single-base resolution using MethylationEPIC BeadChips. QC for the blood DNA methylation is described in detail in [10]. We applied the same QC protocol on the ileal biopsies and retained all 40 ileal biopsies. Data was normalized using the beta-mixture quantile dilation (BMIQ) method [27]. Estimated cell counts for CD4+ T cells, CD8+ T cells, NK cells, B cells, monocytes, and 
granulocytes were calculated using Houseman's approach [28]. After exclusion of CpGs with SNPs in their probe sequences, DNA methylation of 609,192 CpG sites in 402 samples from 238 subjects was used in this study.

Blood genotypes for GTP cohort: Study design and genotyping of the replication cohort was previously described in recent studies [29, 30]. Briefly, GTP individuals were prospectively recruited from waiting rooms at Grady Memorial Hospital in Atlanta, GA between 2005-2008 for a study of stressful life events and symptoms of post-traumatic stress disorder (PTSD). 791 subjects from GTP were genotyped using Illumina HumanOmni1-Quad, HumanOmniExpress or Multi-Ethnic Global arrays. Genotypes were called using Illumina's GenomeStudio software. QC analyses on the genetic data from each chip were done separately, and untyped variants were imputed with IMPUTE2 software [26] using 1000 Genomes Project Phase3 as a reference panel. Individuals with > 5\% missing data were removed. SNPs with > 95\% call rate, MAF $>1 \%$ and Hardy-Weinberg equilibrium (HWE) $P>10^{-5}$ were included. As a result, 5,971,966 genomic variants for 780 samples were retained. We used PLINK software [15] to perform genotype QC in both discovery and replication cohorts.

Blood DNAm for GTP cohort DNA was extracted from whole blood and interrogated using the MethylationEPIC BeadChip according to manufacturer's instructions. Raw methylation beta values from the Infinium MethylationEPIC BeadChip were determined via the Illumina GenomeStudio program. Samples with probe detection call rates $<90 \%$ and those with an average intensity value of either $<50 \%$ of the experiment-wide sample mean or $<2,000$ arbitrary units (AU) were removed using the R package CpGassoc [31]. Data points with detection p-values $>0.01$ were set to missing. CpG sites that crosshybridize between autosomes and sex chromosomes were removed. BMIQ was used to normalize the distribution of types I and II probes [27]. In total, methylation of $608,245 \mathrm{CpG}$ sites was examined in 780 blood samples of the replication cohort. 
mQTL (Methylation Quantitative Trait Loci) study: All SNPs passing QC were tested for association with $\sim 600 \mathrm{~K} \mathrm{CpG}$ sites using a linear mixed model implemented in matrixeQTL [32]. The model was adjusted for age, gender, estimated cell proportions and three genotype-based principal components (PCs) to adjust for population sub-structure, and treated CpG-specific methylation as the outcome and SNP allele count as an explanatory variable. First, we tested each $\mathrm{CpG}$ site against all SNPs for both cis- and trans- SNP-CpG associations in the discovery cohort of 238 samples at diagnosis (164 CD cases and 74 controls), with adjustment for disease status in addition to other covariates. A stringent Bonferroni threshold of $P<8.21 \times 10^{-14}$ was used to identify mQTLs in the CD (discovery) cohort. To replicate our findings, we further tested all significant associations in the GTP cohort using the same model as in the discovery cohort, adjusting for age, gender, PTSD status, and estimated cell proportions from DNA methylation data. To identify whether these associations are specifically observed in the disease- and longitudinal datasets, we tested and compared them in the 74 healthy controls, $164 \mathrm{CD}$ cases at diagnosis and at 3-year follow-up, separately. We also tested the significant blood mQTLs in a cohort of ileal samples ( $n=40$, which is a subset of individuals from the discovery cohort) to assess tissue-specific associations. For these follow-up analyses in ileum, we restricted analysis to SNPs that had at least 2 individuals in each genotype category $(\mathrm{AA} / \mathrm{AB} / \mathrm{BB})$ to ensure sufficient genetic variation. A statistical significance threshold of $P<0.05$ was used for replication analyses of the disease-, longitudinal-, and tissue- associated mQTLs. We used the clump command in PLINK [15] to identify the number of independent associations for each genomic variant with more than 1 significant SNP-CpG association by using default parameters.

Annotation of SNPs and CpG sites: We used the annovar package [33] to annotate SNPs, and the MethylationEPIC annotation file to annotate $\mathrm{CpG}$ sites.

KEGG pathway enrichment analysis: We used missMethyl [34], a R/Bioconductor package, to identify KEGG database pathways that are more likely to occur in the associated CpGs than would be expected by chance. Genes with more $\mathrm{CpG}$ probes on the MethylationEPIC array are more likely to have differentially 
methylated CpGs, which could introduce potential bias when performing pathway enrichment analysis. The gometh function implemented in missMethyl considers the varying number of CpGs per gene by computing prior probability for each gene based on the gene length and the number of CpGs probed per gene on the array.

\section{List of abbreviations:}

Methylation quantitative trait loci (mQTL),

Inflammatory Bowel Disease (IBD),

Crohn's Disease (CD),

Post-traumatic stress disorder (PTSD),

Grady Trauma Project (GTP),

DNA methylation (DNAm),

Genome-wide association studies (GWAS),

Single nucleotide polymorphisms (SNP),

Epigenome-wide association study (EWAS),

Mendelian Randomization (MR),

C-reactive protein (CRP),

Minor allele frequency (MAF),

Hardy-Weinberg equilibrium (HWE),

Linkage disequilibrium (LD),

False Discovery Rate (FDR)

\section{Declarations}

\section{Ethics approval and consent to participate:}

Protocols including signed consent of all participants and/or consent of parents or legal guardians in the case of minors were approved by the IRBs of Emory University for CD RISK study supported by a 
research initiative grant from the Crohn's and Colitis Foundation. The GTP study was approved by the Institutional Review Board of Emory University School of Medicine and the Grady Health Systems Research Oversight Committee. All participants provided written informed consent.

\section{Consent for publication: Not applicable}

Availability of data and materials. The blood DNA methylation data for all the 238 subjects (402 samples) included in this study have been deposited in the Gene Expression Omnibus (GEO) and are accessible through GEO series accession GSE112611. DNA methylation data for the 40 ileal biopsies are also deposited in GEO and can be retrieved through the accession number GSE135905.

\section{Competing interests}

The authors declare no competing financial interests.

\section{Funding}

This work was supported by a research initiative grant from the Crohn's and Colitis Foundation, New York, NY to the individual study institutions participating in the RISK study. This research was also supported by the National Institute of Diabetes and Digestive and Kidney Diseases (NIDDK) of the National Institutes of Health, under grant numbers RO1-DK098231 and RO1-DK087694 to S.K.

\section{Authors Contributions}

S.V., K.N.C., D.J.C., A.K.S. and S.K. conceived of and designed the study. J.P., D.T.O., and S. K., processed samples for methylation profiling and S.V., H.K.S., and V.K. performed the analysis with input from D.J.C. and K.N.C., K.N.C., A.K.S. and S.V., S.K., H.K.S., L.A.D., J.S.H., D.J.C., K.N.C., G.G., A.K.S. and S.K. interpreted the results and wrote the manuscript. All authors reviewed and approved the manuscript prior to submission.

\section{Acknowledgements}


bioRxiv preprint doi: https://doi.org/10.1101/2020.11.16.385534; this version posted November 17, 2020. The copyright holder for this preprint (which was not certified by peer review) is the author/funder. All rights reserved. No reuse allowed without permission.

We are grateful to Anne Dodd, Jason Matthews, and all other RISK investigators for their support and helpful comments. 


\section{Figure legends:}

Fig. 1: Genetic associations with DNAm genome-wide in discovery cohort. (A) In total 535,448 SNPCpG pairs are plotted, including both cis- and trans- associations. The $\mathrm{X}$-axis indicates genomic variant position, whereas the $\mathrm{Y}$-axis indicates $\mathrm{CpG}$ site position. The color of each point represents absolute differences in DNAm per allele relative to the reference allele. Point size represents the significance of the association. A clear positive diagonal line indicates that the majority of associations are cis associations. (B) 498,261 cis associations are plotted by position of CpG relative to SNP. The strongest associations are seen for proximal pairs of SNPs and CpG sites. (C) 37,187 trans- associations are plotted, with most of them occurring within the same chromosome.

Fig. 2: Replication of mQTL associations in replication cohort. Effect sizes for 425,288 SNP-CpG pairs are compared between discovery and replication cohorts. Associations significant in both discovery cohort $\left(P<8.21 \times 10^{-14}\right)$ and replication cohort $\left(P<5 \times 10^{-8}\right)$ are marked in maroon. Gray dots indicate associations significant only in discovery cohort.

Fig. 3: Disease-, disease course- or tissue- specific associations in discovery cohort. For 498,261 cis SNP-CpG pairs significant in the discovery cohort of 238 blood samples, Figure 3 shows effect sizes estimated in the following subgroups: (A) 74 healthy controls vs. $164 \mathrm{CD}$ cases at diagnosis, (B) $164 \mathrm{CD}$ cases at diagnosis vs. their 3-year follow-up, (C) 74 healthy controls at baseline vs. $164 \mathrm{CD}$ cases at follow-up, and (D) all 238 blood samples vs. 40 ileal biopsies. Maroon color indicates the associations that are significant $\left(P<5 \times 10^{-8}\right)$ in both subgroups. Gray indicates the associations that are significant in one subgroup but not the other.

Fig. 4: Comparing CpG-specific associations with IBD SNPs in blood vs. ileum. (A) Frequency of CpG sites that are associated with previously-identified IBD SNPs in discovery cohort at FDR $<0.05$. In total, 454 associations are considered. (B) Effect sizes of 454 SNP-CpG associations compared in CD cases vs. controls in blood. X-axis shows the effect sizes in CD cases $(n=164)$ and $Y$-axis shows the effect sizes for the same associations in controls $(n=74)(C)$ Effect sizes of 370 SNP-CpG associations found in blood are compared to effect sizes observed in ileum. The nominally significant associations in ileum $(P$ $<0.05$ ) are marked in maroon. (D) Effect sizes of 269 SNP-CpG associations compared across the disease groups in ileum. $\mathrm{X}$-axis shows effect sizes in $\mathrm{CD}$ cases $(\mathrm{n}=17)$ and $\mathrm{Y}$-axis shows effect sizes for the same associations in controls $(n=23)$. 
Fig. 5: Testing the effects of CD-associated CpG sites in blood and ileum. (A) Frequency of SNPs that are associated with CD-associated CpG sites in discovery cohort at FDR $<0.05$ are plotted. In total, 7819 associations with $289 \mathrm{CD}$-associated $\mathrm{CpG}$ sites are considered. (B) Effect sizes of 7819 SNP-CpG associations compared in cases vs. controls in blood. $\mathrm{X}$-axis shows the effect sizes in CD cases $(\mathrm{n}=164)$ and $\mathrm{Y}$-axis shows the effect sizes for the same associations in controls ( $\mathrm{n}=74)$ (C) Effect sizes of 6221 SNP-CpG associations from blood are compared to effect sizes observed in ileum. Associations nominally significant in ileum $(P<0.05)$ are marked in maroon. (D) Effect sizes of 4142 SNP-CpG associations compared in cases vs. controls in ileum. X-axis shows effect sizes in CD cases $(n=17)$ and $Y$ axis shows effect sizes for the same associations in controls $(n=23)$. 


\section{References:}

1. Farh KK, Marson A, Zhu J, Kleinewietfeld M, Housley WJ, Beik S, Shoresh N, Whitton H, Ryan RJ, Shishkin AA et al: Genetic and epigenetic fine mapping of causal autoimmune disease variants. Nature 2015, 518(7539):337-343.

2. Farber CR, Lusis AJ: Integrating global gene expression analysis and genetics. Adv Genet 2008, 60:571-601.

3. Rockman MV, Kruglyak L: Genetics of global gene expression. Nat Rev Genet 2006, 7(11):862-872.

4. Ward LD, Kellis M: Interpreting noncoding genetic variation in complex traits and human disease. Nat Biotechnol 2012, 30(11):1095-1106.

5. Venkateswaran S, Marigorta UM, Denson LA, Hyams JS, Gibson G, Kugathasan S: Bowel Location Rather Than Disease Subtype Dominates Transcriptomic Heterogeneity in Pediatric IBD. Cellular and Molecular Gastroenterology and Hepatology 2018, 6(4):474-+.

6. Mo A, Marigorta UM, Arafat D, Chan LHK, Ponder L, Jang SR, Prince J, Kugathasan S, Prahalad S, Gibson G: Disease-specific regulation of gene expression in a comparative analysis of juvenile idiopathic arthritis and inflammatory bowel disease. Genome Med 2018, 10(1):48.

7. Thalayasingam N, Nair N, Skelton AJ, Massey J, Anderson AE, Clark AD, Diboll J, Lendrem DW, Reynard LN, Cordell HJ et al: CD4+and B Lymphocyte Expression Quantitative Traits at Rheumatoid Arthritis Risk Loci in Patients With Untreated Early Arthritis Implications for Causal Gene Identification. Arthritis \& Rheumatology 2018, 70(3):361-370.

8. Handy DE, Castro R, Loscalzo J: Epigenetic modifications: basic mechanisms and role in cardiovascular disease. Circulation 2011, 123(19):2145-2156.

9. Moore LD, Le T, Fan G: DNA methylation and its basic function. Neuropsychopharmacology 2013, 38(1):23-38.

10. Somineni HK, Venkateswaran S, Kilaru V, Marigorta UM, Mo A, Okou DT, Kellermayer R, Mondal K, Cobb D, Walters TD et al: Blood-Derived DNA Methylation Signatures of Crohn's Disease and Severity of Intestinal Inflammation. Gastroenterology 2019, 156(8):2254-2265 e2253.

11. Hannon E, Gorrie-Stone TJ, Smart MC, Burrage J, Hughes A, Bao Y, Kumari M, Schalkwyk LC, Mill J: Leveraging DNA-Methylation Quantitative-Trait Loci to Characterize the Relationship between Methylomic Variation, Gene Expression, and Complex Traits. Am J Hum Genet 2018, 103(5):654-665.

12. Wu Y, Zeng J, Zhang F, Zhu Z, Qi T, Zheng Z, Lloyd-Jones LR, Marioni RE, Martin NG, Montgomery GW et al: Integrative analysis of omics summary data reveals putative mechanisms underlying complex traits. Nat Commun 2018, 9(1):918.

13. Gaunt TR, Shihab HA, Hemani G, Min JL, Woodward G, Lyttleton O, Zheng J, Duggirala A, McArdle WL, Ho K et al: Systematic identification of genetic influences on methylation across the human life course. Genome Biol 2016, 17:61.

14. Kugathasan S, Denson LA, Walters TD, Kim MO, Marigorta UM, Schirmer M, Mondal K, Liu C, Griffiths A, Noe JD et al: Prediction of complicated disease course for children newly diagnosed with Crohn's disease: a multicentre inception cohort study. Lancet 2017, 389(10080):1710-1718. 
15. Purcell S, Neale B, Todd-Brown K, Thomas L, Ferreira MAR, Bender D, Maller J, Sklar P, de Bakker PIW, Daly MJ et al: PLINK: A tool set for whole-genome association and population-based linkage analyses. American Journal of Human Genetics 2007, 81(3):559-575.

16. Almli LM, Lori A, Meyers JL, Shin J, Fani N, Maihofer AX, Nievergelt CM, Smith AK, Mercer KB, Kerley K et al: Problematic alcohol use associates with sodium channel and clathrin linker 1 (SCLT1) in trauma-exposed populations. Addiction Biology 2018, 23(5):1145-1159.

17. de Lange KM, Moutsianas L, Lee JC, Lamb CA, Luo Y, Kennedy NA, Jostins L, Rice DL, Gutierrez-Achury J, Ji SG et al: Genome-wide association study implicates immune activation of multiple integrin genes in inflammatory bowel disease. Nat Genet 2017, 49(2):256-261.

18. Liu JZ, van Sommeren S, Huang H, Ng SC, Alberts R, Takahashi A, Ripke S, Lee JC, Jostins L, Shah T et al: Association analyses identify 38 susceptibility loci for inflammatory bowel disease and highlight shared genetic risk across populations. Nat Genet 2015, 47(9):979-986.

19. Smith AK, Kilaru V, Kocak M, Almli LM, Mercer KB, Ressler KJ, Tylavsky FA, Conneely KN: Methylation quantitative trait loci (meQTLs) are consistently detected across ancestry, developmental stage, and tissue type. BMC Genomics 2014, 15:145.

20. Qi T, Wu Y, Zeng J, Zhang FT, Xue AL, Jiang LD, Zhu ZH, Kemper K, Yengo L, Zheng ZL et al: Identifying gene targets for brain-related traits using transcriptomic and methylomic data from blood. Nature Communications 2018, 9.

21. Zhang B, Zhou Y, Lin N, Lowdon RF, Hong CB, Nagarajan RP, Cheng JB, Li DF, Stevens M, Lee HJ et al: Functional DNA methylation differences between tissues, cell types, and across individuals discovered using the M\&M algorithm. Genome Research 2013, 23(9):1522-1540.

22. Lokk K, Modhukur V, Rajashekar B, Martens K, Magi R, Kolde R, Koltsina M, Nilsson TK, Vilo J, Salumets A et al: DNA methylome profiling of human tissues identifies global and tissue-specific methylation patterns. Genome Biol 2014, 15(4):r54.

23. Sonawane AR, Platig J, Fagny M, Chen CY, Paulson JN, Lopes-Ramos CM, DeMeo DL, Quackenbush J, Glass K, Kuijjer ML: Understanding Tissue-Specific Gene Regulation. Cell Rep 2017, 21(4):1077-1088.

24. Yang RY, Quan J, Sodaie R, Aguet F, Segre AV, Allen JA, Lanz TA, Reinhart V, Crawford M, Hasson S: A systematic survey of human tissue-specific gene expression and splicing reveals new opportunities for therapeutic target identification and evaluation. bioRxiv 2018:311563.

25. Barbeira AN, Dickinson SP, Bonazzola R, Zheng J, Wheeler HE, Torres JM, Torstenson ES, Shah KP, Garcia T, Edwards TL et al: Exploring the phenotypic consequences of tissue specific gene expression variation inferred from GWAS summary statistics. Nat Commun 2018, 9(1):1825.

26. Howie B, Fuchsberger C, Stephens M, Marchini J, Abecasis GR: Fast and accurate genotype imputation in genome-wide association studies through pre-phasing. Nature Genetics 2012, 44(8):955-+.

27. Teschendorff AE, Marabita F, Lechner M, Bartlett T, Tegner J, Gomez-Cabrero D, Beck $\mathrm{S}$ : A beta-mixture quantile normalization method for correcting probe design bias in Illumina Infinium 450 k DNA methylation data. Bioinformatics 2013, 29(2):189-196. 
28. Houseman EA, Accomando WP, Koestler DC, Christensen BC, Marsit CJ, Nelson HH, Wiencke JK, Kelsey KT: DNA methylation arrays as surrogate measures of cell mixture distribution. BMC Bioinformatics 2012, 13:86.

29. Almli LM, Lori A, Meyers JL, Shin J, Fani N, Maihofer AX, Nievergelt CM, Smith AK, Mercer KB, Kerley K et al: Problematic alcohol use associates with sodium channel and clathrin linker 1 (SCLT1) in trauma-exposed populations. Addict Biol 2018, 23(5):11451159.

30. Nievergelt CM, Maihofer AX, Klengel T, Atkinson EG, Chen CY, Choi KW, Coleman JRI, Dalvie S, Duncan LE, Gelernter J et al: International meta-analysis of PTSD genome-wide association studies identifies sex- and ancestry-specific genetic risk loci. Nat Commun 2019, 10(1):4558.

31. Barfield RT, Kilaru V, Smith AK, Conneely KN: CpGassoc: an R function for analysis of DNA methylation microarray data. Bioinformatics 2012, 28(9):1280-1281.

32. Shabalin AA: Matrix eQTL: ultra fast eQTL analysis via large matrix operations. Bioinformatics 2012, 28(10):1353-1358.

33. Wang K, Li M, Hakonarson H: ANNOVAR: functional annotation of genetic variants from high-throughput sequencing data. Nucleic acids research 2010, 38(16):e164-e164.

34. Phipson B, Maksimovic J, Oshlack A: missMethyl: an R package for analyzing data from Illumina's HumanMethylation450 platform. Bioinformatics 2016, 32(2):286-288. 


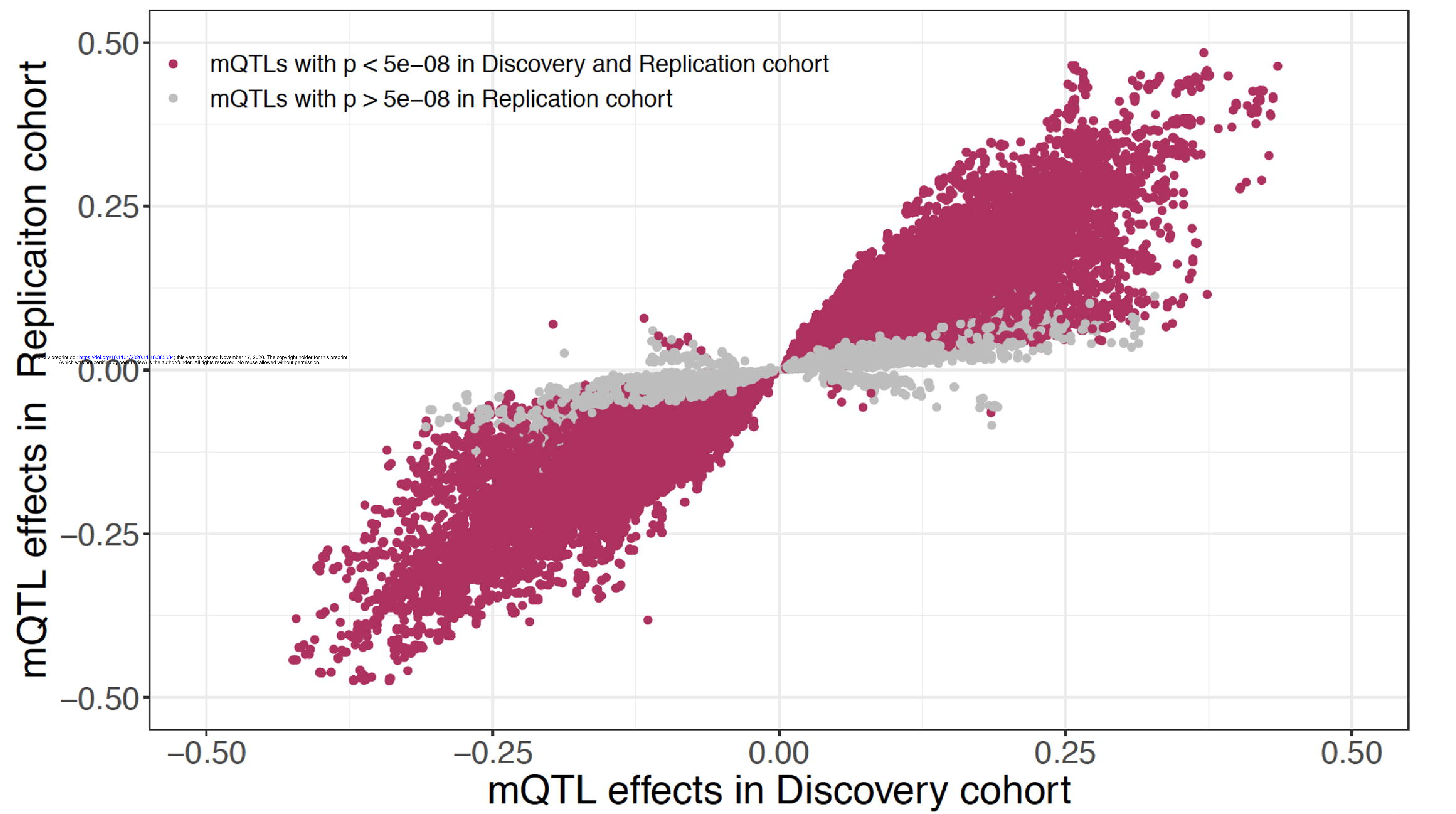

Figure 2 

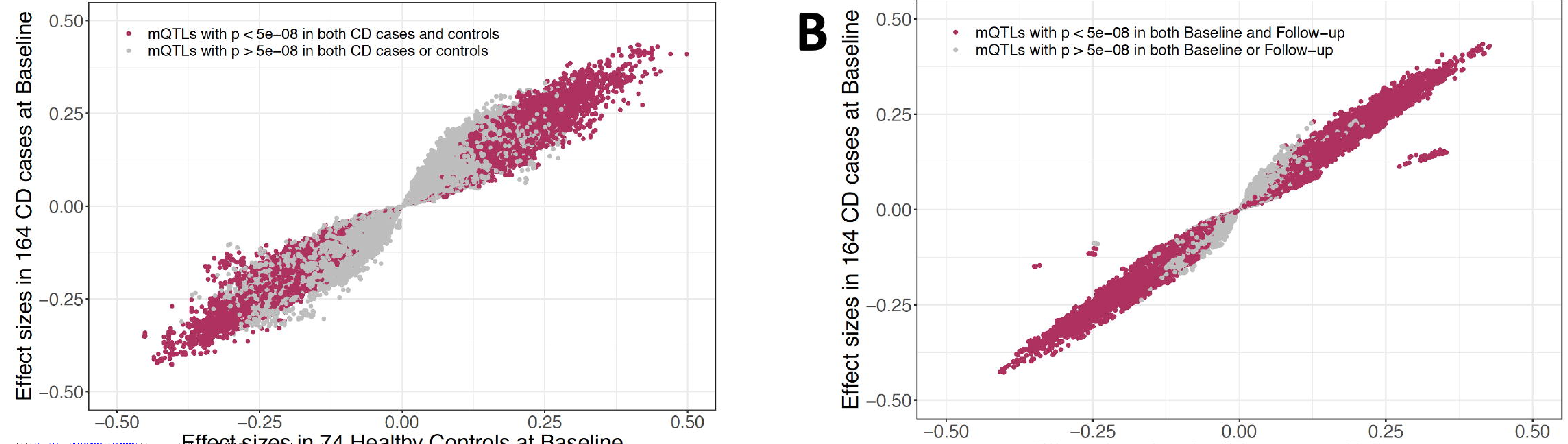

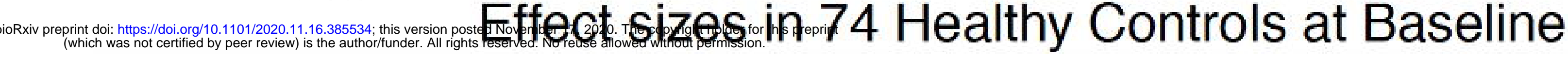
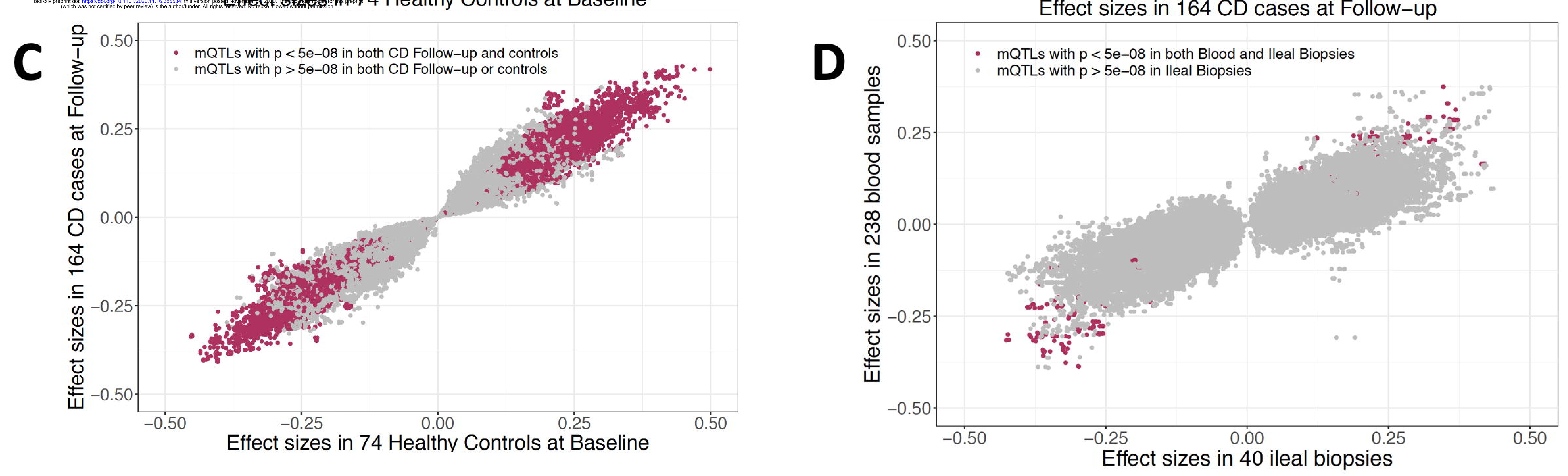

Figure 3 
